\title{
An hybrid neuro-wavelet approach for long-term prediction of solar wind
}

\author{
Christian Napoli ${ }^{1}$, Francesco Bonanno ${ }^{2}$ and Giacomo Capizzi ${ }^{2}$ \\ ${ }^{1}$ Dept. of Physics and Astronomy, University of Catania, \\ Via S. Sofia, 95125, Catania - ITALY \\ email: chnapoli@gmail.com \\ ${ }^{2}$ Dept. of Electrical, Electronic and Systems Engineering, University of Catania, \\ Viale A. Doria, 95125, Catania - ITALY \\ email: gcapizzi@diees.unict.it
}

\begin{abstract}
Nowadays the interest for space weather and solar wind forecasting is increasing to become a main relevance problem especially for telecommunication industry, military, and for scientific research. At present the goal for weather forecasting reach the ultimate high ground of the cosmos where the environment can affect the technological instrumentation. Some interests then rise about the correct prediction of space events, like ionized turbulence in the ionosphere or impacts from the energetic particles in the Van Allen belts, then of the intensity and features of the solar wind and magnetospheric response. The problem of data prediction can be faced using hybrid computation methods so as wavelet decomposition and recurrent neural networks (RNNs). Wavelet analysis was used in order to reduce the data redundancies so obtaining representation which can express their intrinsic structure. The main advantage of the wavelet use is the ability to pack the energy of a signal, and in turn the relevant carried informations, in few significant uncoupled coefficients. Neural networks (NNs) are a promising technique to exploit the complexity of non-linear data correlation. To obtain a correct prediction of solar wind an RNN was designed starting on the data series. As reported in literature, because of the temporal memory of the data an Adaptative Amplitude Real Time Recurrent Learning algorithm was used for a full connected RNN with temporal delays. The inputs for the RNN were given by the set of coefficients coming from the biorthogonal wavelet decomposition of the solar wind velocity time series. The experimental data were collected during the NASA mission WIND. It is a spin stabilized spacecraft launched in 1994 in a halo orbit around the L1 point. The data are provided by the SWE, a subsystem of the main craft designed to measure the flux of thermal protons and positive ions.
\end{abstract}

Keywords. solar wind, magnetic fields, Sun: activity, methods: data analysis

\section{Introduction}

The space environment is a dynamic system for the most part driven by the solar radiation and emission of plasma and particles from the star surface. The main effect of the solar activity is of course the solar wind, the set of emissions which voyage from the sun to us, and that can be measured providing informations on the energy and intensity. Solar emissions and electromagnetic disturbances (which could hamper the communication and satellite network), fast particles and flares bursts reaches Earth in few hours or even minutes from the solar event, as far as big clouds of plasma and charged particles hit the globe in the range of few days, without any early warning. The problem of correct and precise prediction of the solar wind then rises starting from the available space weather data survey. The problem of data prediction can be faced by using soft computing techniques as wavelet decompositions, recurrent neural networks or joint hybrid methods (Capizzi, Bonanno \& Napoli 2010, Goh \& Mandic 2003). 


\section{The basic of wavelet and neural networks theory}

As shown by (Cybenko 1989), a finite weighted sum of continuous discriminating functions $\sigma\left(a_{i}{ }^{T} x+b_{i}\right)$, where $b_{i}$ are real scalar and $a_{i}$ n-dimensional real vectors, is dense in the continuous functions space on $[0,1]^{n}$, so any continuous function $f$ can be approximated by this kind of finite sum. Analogously according to wavelet theory we can state that the finite weighted sum of $\operatorname{det}\left(D_{i}{ }^{1 / 2}\right) \psi\left(d_{i} x-t_{i}\right)$ is dense in the $L^{2}\left(\mathbb{R}^{n}\right)$, where $\left\{d_{i}\right\}$ is the dilatation vector, $\left\{t_{i}\right\}$ the translation vector, $D_{i}=\operatorname{diag}\left(d_{i}\right)$ and $\psi$ the mother wavelet function whose translates and dilates forms the basis for the $L^{2}\left(\mathbb{R}^{n}\right)$ space. This statement shows how the wavelets offer high advantages over other kinds of activation functions. In particular the performance of the wavelet decomposition can be noticed for nonlinear dynamical systems and predictors(Daubechies 1990). The wavelet transform, in fact, packs the energy of the signal, and in turn its relevant information in few significant non zero coefficients reducing the redundancies and showing the intrinsic structure in time and frequencies. The so obtained representation with wavelet decomposition offer advantages while used with neural networks. Typically a neural network is composed by the input and output neural layer and generally at least two hidden layers which are the core of the computational process which will assign a set of weights to the connections between different neurons. As reported in literature, in presence of a temporal memory in the dataset recurrent neural networks are used. The recurrent neural networks have a dynamic structure, which evolves during time because these networks contain feedback lines. In particular to obtain the best performance, even in terms of computational complexity and overall error, these neural networks are trained by an Adaptative Amplitude Real Time Recurrent Learning algorithm.

\section{Proposed data analysis and simulations}

The solar wind speed data were provided by the WIND SWE (Kasper 2006) and their analysis was done by using wavelet decomposition in order to pack the relevant informations in few significant coefficients. The approach lead to design a neural network which adapt himself to the signal through the significant wavelet coefficients set. In order to achieve this goal we used biorthogonal wavelets, (which have more freedom degrees respect the orthonormal ones) as result of a multiple evaluation of behaviors for an extended set of different kind of wavelets. The analysis is mainly based on the RMS performance which leaded to our final choose. Biorthogonal wavelets allow more degrees of freedom respect to traditional orthogonal (and of course the not orthogonal) wavelets, and because these have two different scaling functions, which can generate twin resolution analysis by two different wave functions $\psi$ and $\psi^{\prime}$. The scaling sequence must satisfy the biorthogonality condition and the wavelet coefficients in the general form are $b_{n}=(-1)^{n} a_{N-1-n}^{\prime}$ and $b_{n}{ }^{\prime}=(-1)^{n} a_{M-1-n}$. By using the MATLAB package we developed a series of routines performing a biorthogonal wavelet analysis of the data. The coefficients set resulting from the wavelet analysis was used as input for the designed neural network. A biorthogonal wavelet decomposition was used to extract a shortened number of non-zero coefficients from a signal representative of solar wind velocity sampled trough time. These proposed calculations allowed us to drastically cut down the useful data set. The subbands of the decomposition were used to predict the future values of wavelet coefficients. The correlation between different subbands and temporal trends allow the subdivision of the neural network in serial subnets. In this adaptative conception the network can be modularized in relation with the time bands in order to cut the computational process based on the prediction goal at the selected temporal window. The 


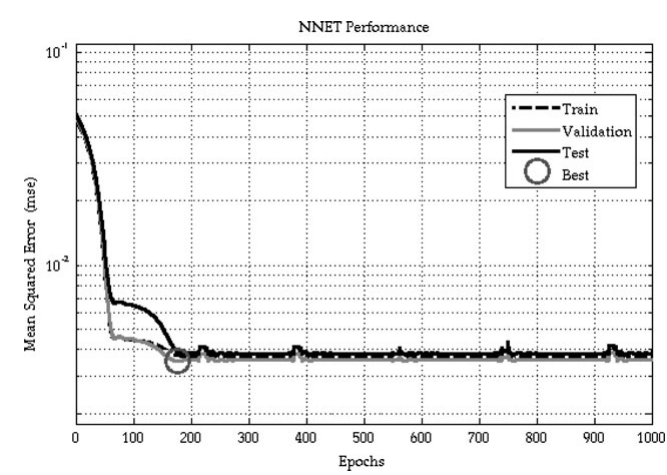

Figure 1. Performance of the NN

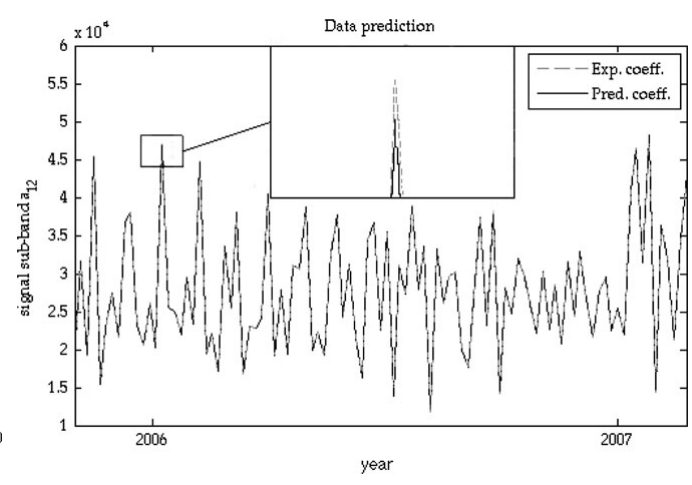

Figure 2. Solar wind velocity prediction

solar wind speed were decomposed in 13 subbands with the described method reducing the $99 \%$ of the energy in less than $4 \%$ of non zero coefficients. We trained the network to the 12 th temporal sub-band to predict the rate of change in the solar wind velocity for the next 6 days. According the proposed modular strategy, for a signal sub band $a_{n}$, and in order to predict the data at a time interval $\tau_{1}$ from a time serie of a previous interval $\tau_{0}$, then the input for the NN is given in the form of $\left[a_{n+1}\left(\tau_{0}\right) \mid d_{n}\left(\tau_{0}\right)\right]$ so that the output will be the sub band $a_{n}\left(\tau_{1}\right)$. The selected NN is a full RNN NARX, trained by a gradient descent with momentum and adaptive amplitude learning rule backpropagation algorithm. The selected NN have 3 hidden layer: the first of 15 neuron with tribas transfer function, the second 11 neurons with tansig transfer function, and the third of 5 neurons with a competitive transfer function. Finally time vectors were used to create 5 delay lines for the input and 3 for the output.

\section{Conclusions and results}

The problem of solar wind data prediction, here faced using hybrid computations methods so as wavelet decomposition and recurrent neural networks, has been demostrated to be efficient. In fact the simulations for long-term prediction of solar wind velocity show the power of the proposed neuro-wavelet method and the obtained good performance in terms of RMS. The predicted solar wind speed accurately matches the experimental data within an RMS of $6 \%$. The performance of the selected neural network is shown in fig. 1 . The most significant results of solar wind velocity prediction are shown in fig. 2 .

\section{References}

Capizzi G., Bonanno F., \& Napoli C. 2010, Proc. Speedam 2010, p. 586

Goh S. L. \& Mandic D. P. 2003, Neural Networks, Vol.16, p. 1095

Cybenko G. 1989, Math. of Control, Signals, and Syst., Vol.2, No.4, p. 303

Daubechies I. 1990, IEEE Trans. Inf. Theory, Vol.36, No.5, p. 961

Kasper J. et al. 2006 J. of Geophys. Res., Vol.1

Gleisner H., Lundstedt H., \& Wintoft P. 1996, Ann. Geophys., Vol.4, No.7, p. 679 